\title{
NUTRITIONAL SUPPORT IN ACUTE SEVERE PANCREATITIS- NASOJEJUNAL VS. NASOGASTRIC FEED
}

\author{
Vikas Garg1, Tejinderpal Singh², P. S. Nain ${ }^{3}$, Anju Bhagtana4, Jyoti Jindal ${ }^{5}$ \\ 1Associate Professor, Department of General Medicine, DMC and H, Ludhiana. \\ 2Senior Resident, Department of General Surgery, Fortis, Ludhiana. \\ 3 Professor, Department of General Surgery, DMC and H, Ludhiana. \\ ${ }^{4}$ Senior Resident, Department of General Surgery, DMC and H, Ludhiana. \\ ${ }^{5}$ Senior Resident, Department of General Medicine, DMC and H, Ludhiana.
}

\begin{abstract}
BACKGROUND

Nutritional support is an important aspect in the management of acute pancreatitis. Enteral feeding can be given either through nasogastric or nasojejunal route. Studies have shown that nasojejunal tube placement is cumbersome and that nasogastric feeding is an effective means of providing enteral nutrition. However, the concern that nasogastric feeding increases the chance of aspiration and exacerbates acute pancreatitis by stimulating pancreatic secretion has prevented it as the standard of care.

The primary objective of this study was to compare nasogastric feeding and nasojejunal feeding routes of enteral nutrition in acute severe pancreatitis with regards to safety and effectiveness.
\end{abstract}

\section{MATERIALS AND METHODS}

This is a Prospective Descriptive study. This study was done to compare nasogastric vs. nasojejunal feeding in acute severe pancreatitis. The total number of patients in the study were sixty, $(n=60)$. Thirty patients were in nasogastric feeding group, while thirty patients were in the nasojejunal group (NG group= 30; NJ group= 30 ).

\section{CONCLUSION}

The nasogastric route of enteral nutrition appears to be an effective route of enteral nutrition in predicted severe acute pancreatitis. Nasogastric feeding is safe and well tolerated, and is simple and easy to establish. The nasogastric route of enteral nutrition appears too comparable to the nasojejunal route in terms of safety, tolerance and efficacy. So both the routes can be used for enteral nutrition in acute severe pancreatitis.

\section{RESULTS}

Serum albumin as measured in biochemical tests was also similar. Serum albumin was measured in all patients at the end of one week and then at the end of 7 weeks after receiving enteral nutrition. Serum albumin had decreased from the baseline. However, the decrease was comparable in both NG and NJ groups and there was no statistically significant difference in both the groups.

\section{KEYWORDS}

Pancreatitis, Nasogastric, Nasojejunal, Feed.

HOW TO CITE THIS ARTICLE: Garg V, Singh T, Nain PS, et al. Nutritional support in acute severe pancreatitis- nasojejunal vs. nasogastric feed. J. Evolution Med. Dent. Sci. 2018;7(05):588-591, DOI: 10.14260/jemds/2018/134

\section{BACKGROUND}

Acute pancreatitis is a common clinical condition of variable severity in which some patients experience mild, self-limited attacks, while others manifest a severe and frequently lethal attack. ${ }^{1}$ Acute pancreatitis is an inflammatory process of the pancreas that involve per pancreatic tissues and remote organ systems. ${ }^{2}$ The incidence of acute pancreatitis has been increasing over recent years. ${ }^{3}$

In cases of acute severe pancreatitis, about $50 \%$ of deaths occur within the first week. They develop an exaggerated systemic inflammatory response syndrome with the development of multiple organ dysfunction syndrome and death. Patients who survive beyond this period often go on to develop extensive retroperitoneal pancreatic necrosis.

'Financial or Other Competing Interest': None.

Submission 21-12-2017, Peer Review 14-01-2018,

Acceptance 20-01-2018, Published 29-01-2018.

Corresponding Author:

Anju Bhagtana,

101 PG Hostel,

DMC\&H,

Ludhiana.

E-mail: anju_bhagtana@rediffmail.com

DOI: $10.14260 /$ jemds $/ 2018 / 134$

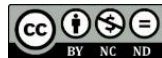

Infection in necrotic tissue leads to sepsis, a persisting systemic inflammatory response and multiple organ dysfunction syndrome and accounts for patient's death. ${ }^{4}$

The pathophysiology of the disease process involves a catabolic stress state, elevated caloric requirement. Reduction in pancreatic stimulation or "pancreatic rest" appeared to be needed to allow resolution of inflammation within the gland. For this reason, acute pancreatitis has been traditionally managed with initial fasting on purpose. ${ }^{5}$ The "pancreatic rest concept" assumes that the pancreatic rest promotes healing, decreases pain and reduces secretion and leakage of the pancreatic juices in pancreatic parenchyma and pancreatic tissue. ${ }^{6}$

Nutritional support is an important aspect in the management of acute pancreatitis. Up to the mid 1990's, total parenteral nutrition had been comprehensively recommended in the acute phase of pancreatitis. ${ }^{7}$ Presently, enteral nutrition has replaced parenteral nutrition.8,9

Enteral nutrition is effective, maintains the intestinal mucosal integrity and reduces infectious and other complications, such as multiple organ deficiency syndrome.10,11,12 Nasojejunal was the established route of enteral nutrition, initially as jejunal feeding does not stimulate pancreatic exocrine secretion. ${ }^{13}$ Recently, 
nasogastric enteral nutrition has been considered in the management of acute pancreatitis, especially severe acute pancreatitis. The Nasogastric route is simple, easy to establish and cost effective. However, this is potentially against to the requirement of pancreatic rest in the acute inflammation phase. Studies indicate nasogastric nutrition to be effective and safe.14,15,16,17 Before recommendation of nasogastric enteral nutrition to clinical practice, further trials are needed. ${ }^{18}$ Hence, the present study was carried out to check the benefit of one route vs. the other.

\section{Aims and Objectives}

The primary objective of this study was to compare Nasogastric feeding and Nasojejunal feeding routes of enteral nutrition in acute severe pancreatitis with regards to safety and effectiveness.

\section{MATERIALS AND METHODS}

This is a Prospective, Descriptive study. A total of 60 consecutive patients admitted to DMC and H, Ludhiana from Sep 2016 to 2017 with objectively graded as acute severe pancreatitis were entered into the study. Randomisation was done by computerised random number generation and the sequence was implemented using numbered containers. Randomised to receive either NG or NJ feeding. This study was done to compare nasogastric vs. nasojejunal feeding in acute severe pancreatitis. Thirty patients were in nasogastric feeding group, while thirty patients were in the nasojejunal group (NG group= 30; $\mathrm{NJ}$ group= 30 ).

\section{Source of Data}

1. Patients admitted in emergency at Dayanand Medical College and Hospital, Ludhiana in the Department of General Surgery during the one year period from Sep 2016 to 2017.

2. A total of 60 patients were admitted in the emergency with the diagnosis of acute severe pancreatitis. 30 patients were included in each group for comparison study.

\section{Statistical Analysis}

The Microsoft version SPSS 17 and chi-square test was used to analyse the data.

\section{RESULTS}

In the present study, the patients taken were 16 years to 65 years of age. The mean age taken in NG group was $37.8 \mathrm{yrs}$. and in NJ group was 40.67 years. The difference in the mean ages taken in the two study groups was statistically not significant.

For this study as mentioned above, 30 patients were taken from NJFT group comprising of $33.3 \%$ females and $66.7 \%$ males, whereas for the NG group same number of patients were selected with $26.67 \%$ females and $73.3 \%$ males. The APACHE II and presence or absence of organ failure was used to assess the severity of acute pancreatitis. The severity was similar in both the NG and NJ groups. At Day 0 RT mean score was at 10.6 and NJFT was 11.40 . On Day 7, the mean score of APACHE II came down with NG at 9 and NJFT at 10.
As per study, organ failure was seen in 8 (53.33\%) patients in NG group and 7 (46.67\%) patients in NJFT group with $p$ value of 0.046 which is statistically significant.

Serum albumin as measured in biochemical tests was also similar. Serum albumin was measured in all patients at the end of one week and then at the end of 7 weeks after receiving enteral nutrition. Serum albumin had decreased from the baseline. However, the decrease was comparable in both NG and NJ groups and there was no statistically significant difference in both groups.

\section{Length of Hospital Stay (LOS)}

Ranged from 8 days to 58 days with a mean of 22.37 days. NG group- range: 2 to 58 days; mean $=22.67$ days. NJ group range: 3 to 29 days; mean $=22.37$ days. There was no significant statistical difference between the two study groups for the length of hospital stay.

\section{ICU Stay}

Ranged from 11 days to 58 days with a mean of 13.60 days. NG group- range: 11 to 58 days; mean $=12.67$ days. NJ grouprange: 11 to 29 days; mean $=14.53$ days. There was no statistical significant difference between the two study groups in total ICU stay.

On comparing the tolerance and side effects, distention and pain/ discomfort during the first 48 hours of initiating feeds were commonly reported. Pain/ discomfort was reported by $33.33 \%$ of patients in the $\mathrm{NG}$ group as compared to $26.67 \%$ in the NJ group.

Distension was seen in $33.33 \%$ in NJ and $33.33 \%$ in NG feed patients after the start of feed, totalling an average of $33.33 \%$ of the total patients taken for this study as shown in the table.

\section{High RT Aspirate}

The high RT Aspirate was present in $33.3 \%$ patients in NJ feed group and in no patient with NG feed group. The $\mathrm{p}$ value was 0.014 .

\section{Feed Tolerance}

Feed intolerance in the two study groups were statistically not significant.

\section{Ventilator Support}

In the study taken up the total 60 patients, 10 patients were on ventilator support, i.e. $20 \%$ (6 patients within the group of 30 ) in the NG group and $13.33 \%$ (4 patients within the group of 30 ) in the $\mathrm{NJ}$ feed group.

Mortality was found to be at $13.33 \%$, specifically in NG feed patients and $26.67 \%$ of the NJFT patients.

On comparison of outcomes between the NG and NJ groups there was no statistical difference in the total length of hospital stay, need for ventilator support, organ failure, and ICU stay between the two groups.

\section{DISCUSSION}

Feeding and Nutritional support form an important aspect in the management of acute pancreatitis. Acute pancreatitis has been traditionally managed with initial fasting on purpose. ${ }^{7}$ The present study was to compare Nasogastric feeding and Nasojejunal feeding routes of enteral nutrition in acute severe 
pancreatitis and to study the effectiveness and safety of these routes of enteral nutrition in acute severe pancreatitis.

In the present study, severe acute pancreatitis was defined in accordance with the APACHE II score and presence or absence of organ failure was used to assess severity. This study was to compare nasogastric and nasojejunal feed in acute severe pancreatitis. Total number of patients in the study were 60 patients. Thirty patients were in the nasogastric feeding group, while thirty patients were in the nasojejunal group. The mean age was 39.27 years with the age range from 16 to 65 years. This was similar to study done by Eatock et al. ${ }^{19}$ The mean age was less compared to the study conducted by Petrov ${ }^{20}$ et al. Most of the patients in this study were 21 males and 9 females, i.e. $70 \%$ males and $30 \%$ females. There was slight male preponderance, which was similar to study done by Eatock et al and Kumar et al.

The aetiology was alcohol in about $46.67 \%$ and biliary in $36.67 \%$, thus the most common aetiology was alcohol induced which was different from study done by Eatock et al in which gallstone was the most common cause i.e. $65.3 \%$. However, the percentage of alcohol in the nasogastric and nasojejunal groups were $33.33 \%$ and $60 \%$ respectively, which was statistically not significant. In the present study, severe acute pancreatitis was defined in accordance with the APACHE II score at admission and presence or absence of organ failure were used to assess severity. The two groups $\mathrm{NG}$ and NJ were similar in these characteristics at the time of admission, which gradually decreased during the first week of illness which was similar to studies done by Eatock et al.

In our study, organ failure was seen in $26.67 \%$ in NG group and $13.3 \%$ in NJFT group which was similar to that of Kumar et al of $18.8 \%$. However, most studies reported a higher percentage of patients requiring ventilator support, but in our study, requirement was statistically not significant in both the NG group and the NJ group.

There was no significant difference in the nutritional parameters in the two groups at baseline and during hospital stay. There was a decline in the nutritional parameters in both groups, as shown by biochemical levels at the end of the first week. This is similar to study done by Petrov et al.

The total length of hospital stay in NJ group ranged from 3 to 29 days and in NG group ranged from 2 to 58 days. The total mean of hospital in the two groups was 13.6 days, which was similar to studies done by Eatock et al in 2000 and 2005.

The ICU stay was more in the NG group. However, this was not statistically significant. In the nasogastric fed patients, overall length of stay in previous studies reported ranging from 7 to 82 days with mean values between 9 to 24 days. This was comparable to the patients receiving nasojejunal feeds (14.53). In the present study, the mean length of stay was 12.67 days in the nasogastric group. In the present study on comparison of outcomes between the NG and NJ groups, there was no statistically significant difference in the total length of hospital stay and ICU stay. Organ failure in the present study was $20 \%$, which was similar to that of Kumar et al of $18.8 \%$.

A total of 4 patients required surgical intervention in the form of necrosectomy, $8(26.67 \%)$ in the NG group and 4 $(13.33 \%)$ in the NJ group. The percentage of patients undergoing surgery was double in the NG group. The need for surgery in the nasogastric group in the present study was different from reports of Kumar et al and Eckerwall et al.
Regarding the side effects and tolerance to feeds, both NG and NJ groups tolerated feeds. On comparing the tolerance and side effects, distension and pain/ discomfort during the first 48 hours of initiating feeds were commonly reported. $33.33 \%$ of patients in NG group and $26.67 \%$ of patients in NJ group had pain abdomen on start of feed. However, this was not statistically significant. This is similar to the findings of Eatock et al in 2005 and Kumar et al in 2006.

Abdominal distension was seen in $33.33 \%$ and $13.33 \%$ patients in NG and NJ group respectively, which was statistically insignificant. This was similar to the study done by Eatock et al in 2005. High RT aspirate was found in around $33.3 \%$ of patients in NG group.

Mortality was $13.33 \%$ in NG group, while in NJ there was $26.67 \%$. This difference was insignificant statistically. The cause of higher mortality in $\mathrm{NJ}$ was probably due to more sick condition of patients at the time of presentation. Overall, mortality was less as compared to most of studies, but was similar to study done by Eckerwall et al in 2006.

Both NG and NJ appeared to tolerate feeds in similar fashion. The target diet was reached in both groups within 3 days in both groups in about $50 \%$ of the patients. Overall, in about $33.3 \%$ of patients in either group the feed had to be stopped due to intolerance. This is similar to previous studies.

\section{CONCLUSION}

The nasogastric route of enteral nutrition appears to be an effective route of enteral nutrition in predicted severe acute pancreatitis. Nasogastric feeding is safe and well tolerated and is simple and easy to establish. The nasogastric route of enteral nutrition appears too comparable to the nasojejunal route in terms of safety, tolerance and efficacy. So both the routes can be used for enteral nutrition in acute severe pancreatitis.

\section{REFERENCES}

[1] O'Reilly DA, Kingsnorth AN. A brief history of pancreatitis. J R Soc Med 2001;94(3):130-2.

[2] Bradley EL. A clinically based classification system for acute pancreatitis. Arch Surg 1993;128(5):586-90.

[3] Imrie CW. Acute pancreatitis: overview. Eur J Gastroenterol Hepatol 1997;9(2):103-5.

[4] Bhatia M, Wong FL, Cao Y, et al. Pathophysiology of acute pancreatitis. Pancreatology 2005;5(2-3):132-44.

[5] Meier R, Beglinger C, Layer P, et al. ESPEN guidelines on nutrition in acute pancreatitis. European society of parenteral and enteral nutrition. Clin Nutr 2002;21(2):173-83.

[6] McClave SA, Chang WK, Dhaliwal R, et al. Nutrition support in acute pancreatitis: a systematic review of the literature. JPEN J Parenter Enteral Nutr 2006;30(2):143-56.

[7] Tesinsky P. Nutritional care of pancreatitis and its complications. Curr Opin Clin Nutr Metab Care 1999;2(5):395-8.

[8] Marik PE, Zaloga GP. Meta-analysis of parenteral nutrition versus enteral nutrition in patients with acute pancreatitis. BMJ 2004;328(7453):1407.

[9] Mitchell RM, Byrne MF, Baillie J. Pancreatitis. Lancet 2003;361(9367):1447-55. 
[10] Al-Omran M, Groof A, Wilke D. Enteral versus parenteral nutrition for acute pancreatitis. Cochrane Database Syst Rev 2003;(1):CD002837.

[11] Alscher KT, Phang PT, McDonald TE, et al. Enteral feeding decreases gut apoptosis, permeability, and lung inflammation during murine endotoxemia. Am J Physiol Gastrointest Liver Physiol 2001;281(2):G56976.

[12] Hadfield RJ, Sinclair DG, Houldsworth PE, et al. Effects of enteral and parenteral nutrition on gut mucosal permeability in the critically ill. Am J Respir Crit Care Med 1995;152(5 Pt 1):1545-8.

[13] Vu MK, van der Veek PP, Frolich M, et al. Does jejunal feeding activate exocrine pancreatic secretion? Eur J Clin Invest 1999;29(12):1053-9.

[14] García de Casasola G, Cacho Acosta G, Gonzálvez-Gasch A, et al. Nasogastric enteral nutrition in severe acute pancreatitis. Med Clin (Barc) 2008;130(13):492-3.

[15] Kumar A, Singh N, Prakash S, et al. Early enteral nutrition in severe acute pancreatitis: a prospective randomized controlled trial comparing nasojejunal and nasogastric routes. J Clin Gastroenterol 2006;40(5):431-4.
[16] Eatock FC, Chong P, Menezes N, et al. A randomized study of early nasogastric versus nasojejunal feeding in severe acute pancreatitis. Am J Gastroenterol 2005;100(2):432-9.

[17] Eckerwall GE, Tingstedt BB, Bergenzaun PE, et al. Immediate oral feeding in patients with mild acute pancreatitis is safe and may accelerate recovery-a randomized clinical study. Clin Nutr 2007;26(6):75863.

[18] Jiang K, Chen XZ, Xia Q, et al. Early nasogastric enteral nutrition for severe acute pancreatitis: a systematic review. World J Gastroenterol 2007;13(39):5253-60.

[19] Eatock FC, Brombacher GD, Steven A, et al. Nasogastric feeding in severe acute pancreatitis may be practical and safe. Int J Pancreatol 2000;28(1):23-9.

[20] Petrov MS, Correia MI, Windsor JA. Nasogastric tube feeding in predicted severe acute pancreatitis. A systematic review of the literature to determine safety and tolerance. JOP 2008;9(4):440-8. 